\title{
DIFERENCIAS DE ESTRÉS Y AFRONTAMIENTO DEL MISMO SEGÚN EL GÉNERO Y CÓMO AFECTA AL RENDIMIENTO ACADÉMICO EN ESTUDIANTES UNIVERSITARIOS
}

\author{
Josep Vidal-Conti \\ Adrià Muntaner-Mas \\ Pere Palou Sampol \\ Universitat de les Illes Balears
}

RESUMEN: La actual evidencia científica presenta diversos posicionamientos en la definición de los niveles de estrés en función del género y su influencia en el rendimiento académico. Los objetivos del estudio son: a) examinar las diferencias de género en el nivel de estrés académico, de estresores académicos y de estrategias de afrontamiento en estudiantes universitarios; b) evaluar la relación entre los niveles de estrés y el rendimiento académico. Participaron 162 estudiantes universitarios del Grado en Educación Primaria con una edad media de 23.7 ( $\mathrm{DE}=5.68$ ). El instrumento de medida utilizado fue el Inventario SISCO del estrés académico. También se recogió la nota media de expediente académico de los estudios de grado. Los principales resultados fueron: 1) El $96.1 \%$ de las mujeres y el $88.2 \%$ de los hombres presentan niveles de estrés académico; 2) "Las evaluaciones de los profesores" y "el tipo de trabajo que piden los profesores" son los estímulos estresores que muestran una diferencia estadísticamente significativa, percibiendo mayor estrés las mujeres, $(p=0.008$, $p=0.005$; respectivamente); 3) Las mujeres utilizan más "la ventilación y confidencias" como estrategia de afrontamiento, respecto al género masculino $(p=0.001)$; 4) No se halló relación entre estrés y rendimiento académico.

PALABRAS CLAVE: Estudiantes universitarios, estrés académico, diferencias de género, rendimiento académico.

\section{DIFFERENCES OF STRESS AND COPING ACCORDING TO GENDER AND HOW IT AFFECTS THE ACADEMIC PERFORMANCE OF UNIVERSITY STUDENTS}

\footnotetext{
ABSTRACT: Current scientific evidence shows different stances on the determination of the gender differences in academic stress and performance
} 
among university students. The aims of this study are: a) explore gender differences in academic stress, academic stressors and coping strategies among university students; b) assess the relationship between academic stress and academic performance. The participants were 162 university students of the Degree in Primary Education, mean age 23.7 (DE=5.68). The outcomes were assessed using SISCO inventory for academic stress. The academic achievement was determined by the student's grade. The main findings were: 1) $96.1 \%$ and $88.2 \%$ of the female and male students respectively had academic stress; 2 ) "Teachers evaluation" and "the kind of the academic task" were the marked stressors significantly higher in females, $(p=0.008, p=0.005$; respectively); 3 ) Stress coping strategies compared between male and female revealed significant differences in particular the "ventilation and confidences" $(p=0.001) ; 4)$ There was no correlation between stress level and academic performance.

KEYWORDS: Undergraduate students, stress variables, gender differences, academic performance.

Recibido: $12 / 07 / 2017$

Aceptado: 29/10/2017

Correspondencia: Josep Vidal-Conti, Universitat de les Illes Balears, Cra. de Valldemossa, km 7.5, Palma (Illes Balears). Email: josep.vidal@uib.es.

\section{INTRODUCCIÓN}

A lo largo de la historia se ha utilizado el concepto "estrés" para denominar realidades distintas, evidenciando la complejidad del mismo. Generalmente el estrés se ha entendido como una sobrecarga para el individuo, y según la Sociedad Española de para el Estudio de la Ansiedad y el Estrés (http://www.ansiedadyestres.org/) se interpreta como un proceso interactivo, en el cual están en juego las demandas de la situación y los recursos del individuo para afrontar la situación. Las demandas de la situación dependen de la valoración subjetiva que el individuo realiza sobre cómo dicha situación afectará a sus intereses. Un nivel moderado de estrés, es algo normal y consustancial con la vida misma. El ser humano y, en general, lo seres vivos tienen que adaptarse a múltiples situaciones. El proceso de adaptación a las demandas de una situación, pone en marcha normalmente un proceso de estrés, en el que el individuo puede no tener suficientes recursos para afrontar las demandas de la situación.

Según la fuente documental consultada algunos autores distinguen entre estrés positivo y estrés negativo, no obstante podemos considerar que en cualquier caso el estrés continuado produce fatiga y efectos fisiológicos sobre el organismo. Alguno de estos efectos podrían ser trastornos psicofisiológicos (p.e. trastornos gastrointestinales, dolor de espalda, disfunciones sexuales, etc.) y mentales (p.e. depresión, ansiedad, etc.), además del deterioro en el funcionamiento normal del organismo (p.e. déficits de atención y concentración, bajo rendimiento académico, escasa productividad, etc.) (Selye, 1946). 
De entre las diferentes clasificaciones que podemos encontrar del estrés destaca la que toma como referencia el aspecto traumático como línea de base. Así, podemos hablar de estrés sexual, estrés amoroso y marital, estrés por duelo, estrés familiar, estrés académico, estrés médico, estrés militar, estrés ocupacional, y estrés por tortura y encarcelamiento (Orlandini, 1999). En el presente estudio la atención se centra en el estrés académico.

Podemos decir que existe prácticamente unanimidad al afirmar que el estrés forma parte de la vida cotidiana de los estudiantes y que tiene un efecto potencialmente negativo sobre el aprendizaje y el rendimiento académico (Struthers, Perry y Menec, 2000). En concreto, Lumley y Provenzano 2003 (en Cabanach, R., FernándezCervantes, R., González, L., y Freire, C., 2010) afirman que el estrés puede afectar al correcto funcionamiento académico del estudiante universitario, interfiriendo en comportamientos de adaptación tales como la asistencia a las clases, la dedicación al estudio o dificultando procesos cognitivos como son la concentración y la atención.

De manera habitual se utiliza el término estrés académico con relativa facilidad, no obstante su verdadero alcance y limitaciones son aún desconocidos. Tal es el caso del concepto del estrés que "adolece de la ambigua bendición de ser demasiado bien conocido y demasiado poco entendido" (Travers y Cooper, 1997 en Barraza, 2007 p. 27). Esta cita referida al estrés en general es perfectamente aplicable al estrés académico.

Siguiendo a Barraza (2007), "el estrés académico es un proceso sistémico de carácter adaptativo y esencialmente psicológico, que se presenta de manera descriptiva en tres momentos":

(1) El alumno se ve sometido, en contextos escolares, a una serie de demandas que, bajo la valoración del propio alumno, son consideradas estresores (input).

(2) Esos estresores provocan un desequilibrio sistémico (situación estresante) que se manifiesta en una serie de síntomas (indicadores del desequilibrio).

(3) Ese desequilibrio sistémico obliga al alumno a realizar acciones de afrontamiento (output) para restaurar el equilibrio sistémico.

A partir de esta descripción se reconocen tres componentes sistémico-procesuales del estrés académico: estímulos estresores (input), síntomas (indicadores de desequilibrio sistémico) y estrategias de afrontamiento (output). A partir de estos componentes del estrés académico se elaboró el Inventario SISCO del Estrés Académico, utilizado en el presente estudio.

Por otro lado, cabe destacar el aumento experimentado en los últimos años de estudios sobre el estrés del docente; hecho que contrasta con la baja existencia de estudios relacionados con el estrés del estudiante. A excepción de los estudios que analizan la ansiedad estudiantil ante los exámenes y el estrés académico asociado a la evaluación, cabe señalar que un estudio de revisión indica que esta población no ha sido centro de atención en la investigación sobre estrés (Michie, Glachan y Bray, 2001 en González Cabanach et al., 2010). No obstante, existe un determinado número de estudios que, tomando como referente los modelos del ámbito organizacional, tratan de abordar dicha problemática (Chambel y Curral, 2005). Además, el estudio del burnout o el síndrome de "estar quemado" en el trabajo también se ha aplicado a muestras preocupacionales, bajo la denominación de burnout académico (SalanovaSoria et al., 2005). 
De entre los estresores relacionados con el proceso de enseñanza-aprendizaje, se ha hecho especial mención al papel que desempeñan las relaciones sociales establecidas entre los individuos que forman parte de un mismo grupo y especialmente a las de los estudiantes entre sí. El docente define las expectativas y demandas que configuran los roles académicos; el alumnado establece entre sí relaciones y forma grupos humanos, en parte condicionado por el estudio, en parte como fruto del contacto humano y buscan su desarrollo académico en el interior de las aulas. Podríamos decir que la capacidad del alumnado de gestionar sus recursos personales y sociales va a ser determinante en el éxito académico, así como favorecer un mayor aprovechamiento del proceso de aprendizaje, un mejor rendimiento e incluso un mejor pronóstico de futura inserción laboral. En varios modelos explicativos del estrés, la participación en la toma de decisiones y el apoyo social de profesores y compañeros son factores que facilitan una reducción del estrés, de ahí la importancia de un buen clima social (Cabanach et al., 2010). Así, el apoyo social contribuye a que el estudiante afronte de manera más eficaz la situación estresante o bien reduce la experiencia de estrés cuando ya ésta se ha producido (Ojeda Pérez, Ramal López, Calvo Francés y Vallespín Montero, 2001).

Uno de los factores que están más relacionados con la aparición del estrés en el contexto académico es la sobrecarga de trabajo, la cual cabe diferenciarse entre el exceso de trabajo (sobrecarga cuantitativa) y la excesiva dificultad y complejidad del mismo (sobrecarga cualitativa). Como consecuencia de la limitación del tiempo disponible para dedicar a los estudios, de las propias limitaciones o como resultado de un plan de estudio con un número excesivo de asignaturas y créditos o con un grado de exigencias excesivamente elevado, entre otras motivos, los estudiantes se ven sobrepasados en sus recursos (Cabanach et al., 2010).

En muchos estudios se ha identificado una interacción entre la ansiedad y situaciones de estrés de evaluación sobre el rendimiento académico en universitarios (Keogh, Bond y Flaxman, 2006; Sohail, 2013). Concretamente, en el área de rendimiento académico, un alto nivel de estrés altera el triple sistema de respuestas: (a) a nivel cognitivo, los alumnos creen no saber nada antes del examen, que van a perder la beca, que se les va a preguntar justo las cuestiones que no se han preparado, si fracasan en el examen que se les va a quedar la mente en blanco, etc., (b) a nivel motor, los alumnos se muerden las uñas, necesitan ir al baño sin eliminación urinaria, emiten resoplos y bufidos, se crujen los dedos, fuman en exceso, etc., y (c) a nivel fisiológico, sufren sudoración, taquicardia, temblor generalizado, diarrea, meteorismo, etc. La alteración en estos tres niveles de respuestas influye de manera directa y negativa en el rendimiento académico, y en algunos casos disminuye la calificación de los alumnos en los exámenes y, en otros casos, los alumnos no llegan a presentarse al examen o abandonan el aula o antes de dar comienzo el examen (Pérez, Martín, Rodríguez, Borda y Del Río, 2003).

La aparición del Espacio Europeo de Educación Superior (EEES) dio lugar a un antes y un después en el proceso de enseñanza-aprendizaje, el cual debería estar asociado a una mayor satisfacción por parte del alumnado, dado que adquiere un rol más activo y partícipe del proceso, con una mejor distribución de las cargas de trabajo y un proceso de tutorización más cercano. Estos hechos que podrían asociarse a una disminución de los niveles de estrés respecto a sistemas de enseñanza anteriores 
no se ven reflejados y sin embargo, con el EEES se han visto incrementadas las cargas de trabajo así como una distribución de las mismas a lo largo del curso, de manera que la dedicación del alumnado a sus estudios durante el curso académico es máxima. Estos hechos por tanto, podrían asociarse a un aumento de los niveles de estrés.

Los altos índices de prevalencia de síntomas de estrés entre los estudiantes universitarios es alarmante (Bayram y Bilgel, 2008). Algunos estudios epidemiológicos confirman que las estudiantes universitarias presentan mayores niveles de estrés percibido que los hombres, (Backovi, Živojinovi, Maksimovi y Maksimovi, 2012; Ekpenyong, Daniel y Aribo, 2013), aunque otros autores no han encontrado diferencias significativas por género (Lue, Chen, Wang, Cheng y Chen, 2010). Escasa es la evidencia que analiza la percepción de los estresores y las estrategias de afrontamiento de éstos en función del género (Hamaideh, 2012), siendo ésta llevada a cabo mediante muestras de población distintas a la del presente estudio. La misma controversia es encontrada cuando se analiza la relación entre estrés académico y rendimiento académico (Park et al., 2012; Sohail, 2013). Las intervenciones para reducir el estrés en estudiantes universitarios son eficaces (Regehr, Glancy y Pitts, 2013), pero para su diseño es necesario conocer los principales estresores, las estrategias de afrontamiento y la relación del estrés con el rendimiento académico.

El presente estudio se Ilevó a cabo en la Universitat de les Illes Balears con alumnos de los estudios de Grado en Educación Primaria. Estos estudios se iniciaron en el curso 2009-10 en sustitución de las titulaciones de maestro de primaria y de las especialidades de educación musical, educación física, lengua extranjera y educación especial. La finalidad de esta etapa es la contribución al desarrollo personal, intelectual y social de la población escolar, mediante los procesos de enseñanza-aprendizaje de contenido científico, matemático, lingüístico, social, ético, artístico, psicomotor y tecnológico, dado que en relación a la profesión de maestro, la sociedad actual demanda un profesional cualificado, que no solamente sepa enseñar conceptos, sino que, con su forma de enseñar, transmita unos valores y unas actitudes.

Los objetivos del estudio eran: a) examinar las diferencias de género en el nivel de estrés académico, en los estresores académicos y en las estrategias de afrontamiento en estudiantes universitarios; b) evaluar la relación entre los niveles de estrés y el rendimiento académico.

\section{Método}

\section{Participantes}

La población objeto de estudio fueron todos alumnos del Grado en Educación Primaria de la Universitat de les Illes Balears. El estudio se llevó a cabo con una muestra final de 162 participantes, de los cuales 34 fueron hombres (21\%) y 128 mujeres $(79 \%)$, con una edad media de 23.7 años (DE=5.68). La muestra se obtuvo por muestreo incidental.

\section{Instrumentos}

El instrumento de medida utilizado para la recogida de datos fue el Inventario SISCO del estrés académico (Barraza, 2007), del cual se administraron dos dimensiones 
(estresores y estrategias de afrontamiento) cada una de ella compuesta por nueve y ocho ítems respectivamente puntuados sobre una escala de tipo Likert con 5 opciones de respuesta en función de la intensidad. Por otro lado se recogió información relativa al género, edad y rendimiento académico (nota media de expediente del grado en el momento de la recogida de datos).

\section{Procedimiento}

La recogida de datos se realizó mediante el envío de un correo electrónico a través del aula virtual a todo el alumnado matriculado en los estudios de Grado en Educación Primaria. La participación fue voluntaria y se informó de las características y objetivos del estudio. El momento de la toma de datos fue el mismo en todos los grupos, al finalizar el segundo semestre.

\section{Análisis estadístico}

Todos los análisis fueron realizados mediante el paquete estadístico SPSS-21 para Windows, y fueron estratificados por género. Para los análisis descriptivos se utilizaron tablas de contingencia por género y estrés académico, mostrando los porcentajes intragrupo. Para la comparación de variables cualitativas se utilizó la prueba de chicuadrado y para las variables continuas ANOVA de un factor (género). Para identificar la influencia del género y el estrés sobre el expediente académico se realizó una ANOVA de dos factores.

\section{Resultados}

Los resultados obtenidos a partir de los datos del cuestionario utilizado muestran que el 94.4\% del alumnado del Grado en Educación Primaria de la Universitat de les Illes Balears afirma haber padecido situaciones de estrés académico. Las diferencias entre género son notables, $96.1 \%$ de las mujeres frente al $88.2 \%$ de los hombres, aunque no significativas ( $p=.075$ ) (Tabla 1 ).

De entre los participantes que afirmaron haber padecido estrés se cuantificó en una escala de 1 a 5 el nivel de intensidad del mismo, siendo 1 el nivel más bajo y 5 el más alto, obteniendo un valor de medio de 3.86 ( $\mathrm{DE}=.871)$. En el caso de los hombres el valor medio se situó en 3.37 ( $\mathrm{DE}=1.07)$ frente al $3.98(\mathrm{DE}=.78$ ) de las mujeres, estableciéndose diferencia significativas $(p=.006)$. Al analizar porcentualmente cada uno de los niveles de estrés se corrobora y pone de manifiesto que las mujeres presentan niveles de estrés más elevados que los hombres. En los niveles 1 y 2 de intensidad de estrés se situaron el $13.4 \%$ de los hombres y el $3.2 \%$ de las mujeres; en el nivel 3 el $46.7 \%$ de los hombres y el $21.6 \%$ de las mujeres; en los niveles 4 y 5 el $40 \%$ de los hombres y el $75.2 \%$ de las mujeres (Tabla 1 ).

De la dimensión estresores (Tabla 2), destacar que las situaciones del contexto escolar percibidas por los participantes como más estresantes fueron la "sobrecarga de tarea y trabajos escolares" $(\chi=4.06)$ y "las evaluaciones de los profesores" $(\chi=3.95)$. Por otro lado, las menos estresantes fueron "competencia con compañeros de grupo" $(\chi=2.31)$ y "participación en clase" $(\chi=2.45)$. 
Tabla 1. Niveles de estrés académico en función del género

\begin{tabular}{llllrrr}
\hline & Total (n) & Hombres (n) & Mujeres (n) & $\boldsymbol{X}^{2}$ & \multicolumn{1}{c}{$\boldsymbol{g l}$} & Sign. \\
\hline Presentan estrés & $94.4 \%(153)$ & $88.2 \%(30)$ & $96.1 \%(123)$ & 3.162 & 1 & 0.075 \\
\hline Nivel estrés & & & & & \\
1 & $1.3 \%(2)$ & $6.7 \%(2)$ & $0 \%(0)$ & & & \\
2 & $3.9 \%(6)$ & $6.7 \%(2)$ & $3.2 \%(4)$ & & & \\
3 & $26.5 \%(41)$ & $46.7 \%(14)$ & $21.6 \%(27)$ & 19.390 & & \\
4 & $44.5 \% 69)$ & $23.3 \%(7)$ & $49.6 \%(62)$ & & & \\
5 & $23.9 \%(37)$ & $16.7 \%(5)$ & $25.6 \%(32)$ & & & \\
\hline
\end{tabular}

En función del género se identifican diferencias significativas $(p<.05)$ en los estresores de "las evaluaciones de los profesores" ( $F=7.26, p=.008)$, "tipo de trabajo que piden los profesores" ( $F=8.07, p=.005)$, "tiempo limitado para hacer el trabajo" $(F=4.24, p=.041)$. En todos ellos las mujeres presentan niveles de estrés superiores a los hombres.

Tabla 2. Dimensión estresores en función del género

\begin{tabular}{lccccc}
\hline \multicolumn{1}{c}{ Dimensión estresores } & Total & $\begin{array}{c}\text { Hombres } \\
\overline{\mathrm{X}}(\sigma)\end{array}$ & $\begin{array}{c}\text { Mujeres } \\
\overline{\mathrm{X}}(\sigma)\end{array}$ & $F$ & Sign. \\
\hline $\begin{array}{l}\text { Competencia con compañeros de } \\
\text { grupo }\end{array}$ & $2.31(1.13)$ & $2.33(1.12)$ & $2.30(1.14)$ & .016 & .899 \\
$\begin{array}{l}\text { Sobrecarga de tareas y trabajos } \\
\text { escolares }\end{array}$ & $4.06(.80)$ & $3.90(.76)$ & $4.10(.81)$ & 1.458 & .229 \\
Personalidad y carácter del profesor & $3.05(.95)$ & $3.03(1.24)$ & $3.06(.87)$ & .014 & .907 \\
$\begin{array}{l}\text { Las evaluaciones de los profesores } \\
\text { Problemas con el horario de clases }\end{array}$ & $3.95(.88)$ & $3.57(.97)$ & $4.04(.84)$ & 7.264 & .008 \\
$\begin{array}{l}\text { Tipo de trabajo que piden los } \\
\text { profesores }\end{array}$ & $3.12(1.17)$ & $2.77(1.33)$ & $2.71(1.14)$ & .057 & .812 \\
$\begin{array}{l}\text { No entender los temas que se } \\
\text { abordan en clase }\end{array}$ & $2.47(1.06)$ & $2.27(1.01)$ & $2.52(1.07)$ & 1.373 & .243 \\
Participación en clase & $2.45(1.11)$ & $2.40(1.07)$ & $2.46(1.13)$ & .080 & .778 \\
Tiempo limitado para hacer el trabajo & $3.29(1.07)$ & $2.93(.91)$ & $3.38(1.09)$ & 4.237 & .041 \\
\hline Total (sumscore) & $3.04(.55)$ & $2.87(.56)$ & $3.08(.54)$ & 3.544 & .062 \\
\hline
\end{tabular}

De la dimensión estrategia de afrontamiento (Tabla 3), destacar que las acciones que manifestaron que realizan los participantes para restaurar el equilibrio sistémico provocado por los estresores fueron, sobretodo, "concentrarse en resolver la situación que me preocupa" $(\chi=3.82)$ y "elaboración de un plan y ejecución de sus tareas" $(\chi=3.64)$. Por otro lado, las acciones más infrecuentes fueron "la religiosidad" $(\chi=1.30)$ y "elogios a sí mismo" $(\chi=2.26)$. 
En función del género únicamente se identifican diferencias significativas en la acción "ventilación y confidencias" ( $F=11.01, p=.001)$ que las mujeres puntúan 3.61 frente al 2.90 en el caso de los hombres.

Tabla 3. Dimensión estrategias de afrontamiento en función del género

\begin{tabular}{|c|c|c|c|c|c|}
\hline $\begin{array}{c}\text { Dimensión estrategias de } \\
\text { afrontamiento }\end{array}$ & $\begin{array}{l}\text { Total } \\
\bar{X}(\sigma)\end{array}$ & $\begin{array}{l}\text { Hombres } \\
\overline{\mathbf{X}}(\sigma)\end{array}$ & $\begin{array}{c}\text { Mujeres } \\
\overline{\mathbf{X}}(\sigma)\end{array}$ & $\boldsymbol{F}$ & Sign. \\
\hline Habilidad asertiva & $3.51(1.02)$ & $3.57(.63)$ & $3.49(1.09)$ & .129 & .719 \\
\hline $\begin{array}{l}\text { Elaboración de un plan y ejecución } \\
\text { de sus tareas }\end{array}$ & $3.64(.97)$ & $3.43(.86)$ & $3.69(.99)$ & 1.754 & .187 \\
\hline $\begin{array}{l}\text { Concentrarse en resolver la situación } \\
\text { que me preocupa }\end{array}$ & $3.82(.81)$ & $3.97(.62)$ & $3.78(.85)$ & 1.233 & .268 \\
\hline Elogios a sí mismo & $2.26(.93)$ & $2.43(1.01)$ & $2.22(.91)$ & 1.308 & .255 \\
\hline La religiosidad & $1.30(.72)$ & $1.40(.72)$ & $1.27(.72)$ & .759 & .385 \\
\hline $\begin{array}{l}\text { Búsqueda de int } \\
\text { situación }\end{array}$ & $2.59(1.08)$ & $2.43(1.01)$ & $2.62(1.10)$ & .753 & .387 \\
\hline $\begin{array}{l}\text { tratar de obtener lo positivo } \\
\text { ación que preocupa }\end{array}$ & $3.14(.89)$ & $3.07(.87)$ & $3.16(.90)$ & .263 & .609 \\
\hline $\begin{array}{l}\text { Ventilación y confidencias } \\
\text { (verbalización de la situación que } \\
\text { preocupa). }\end{array}$ & $3.47(1.08)$ & $2.90(1.03)$ & $3.61(1.05)$ & 11.012 & .001 \\
\hline Total (sumscore) & $2.97(.44)$ & $2.90(.42)$ & $2.99(.44)$ & 1.077 & .301 \\
\hline
\end{tabular}

Por otro lado, en este estudio se pretendía analizar la relación entre el estrés académico y el rendimiento académico, éste último valorado de 0 a 10 mediante la media de expediente académico del grado en curso. Los resultados muestran que no hay diferencias significativas $(F=1.717, p=.192)$ en el expediente académico entre aquellos participantes que afirmaron haber padecido estrés $(\chi=7.18)$ y aquellos que no $(\chi=7.46)$.

Si analizamos los niveles de estrés y rendimiento académico observamos que tampoco existen diferencias significativas entre ambos $(F=.746, p=.562)$. Aquellos participantes que puntuaron su nivel de estrés como 0 tenían una media de 7.51 puntos, los de nivel 1 un 7.48, los de nivel 2 un 7.42, de nivel 3 un 7.10, de nivel 4 un 7.15 , y de nivel 5 un 7.28 (Tabla 4).

Tabla 4. Efectos de la interacción de género y estrés sobre rendimiento académico

\begin{tabular}{lllllll}
\hline & SC & gl & MC & $\boldsymbol{F}$ & Sig. & TE \\
\hline Género & .132 & 1 & .132 & .335 & .563 & .002 \\
Nivel estrés & 1.549 & 5 & .310 & .785 & .562 & .025 \\
Género x nivel estrés & 1.414 & 4 & .353 & .895 & .469 & .023 \\
Error & 59.627 & 146 & .401 & & & \\
\hline
\end{tabular}

Note: $\mathrm{SC}=$ suma de cuadrados, $\mathrm{gl}=$ grados de libertad, $\mathrm{MC}=$ media cuadrática. 
Al analizar el efecto de la interacción entre género y nivel estrés sobre el rendimiento académico se observa que no es significativo (Tabla 5).

Tabla 5. Diferencias de género en rendimiento académico en función del nivel de estrés

\begin{tabular}{cccc}
\hline Nivel estrés & $\begin{array}{c}\text { Total } \\
\overline{\mathbf{X}}(\boldsymbol{\sigma})\end{array}$ & $\begin{array}{c}\text { Hombres } \\
\overline{\mathbf{X}}(\boldsymbol{\sigma})\end{array}$ & $\begin{array}{c}\text { Mujeres } \\
\overline{\mathbf{X}}(\boldsymbol{\sigma})\end{array}$ \\
\hline 0 & $7.51(.45)$ & $7.64(.24)$ & $7.34(.65)$ \\
1 & $7.48(1.13)$ & $7.48(1.13)$ & -- \\
2 & $7.42(.45)$ & $6.92(.06)$ & $7.67(.29)$ \\
3 & $7.10(.71)$ & $6.97(.73)$ & $7.17(.70)$ \\
4 & $7.15(.56)$ & $7.31(.44)$ & $7.13(.58)$ \\
5 & $7.28(.66)$ & $7.26(.69)$ & $7.28(.67)$ \\
\hline
\end{tabular}

\section{Discusıón}

Los resultados de nuestro estudio muestran cuáles son los niveles de estrés del alumnado universitario, concretamente de los estudios de Grado en Educación Primaria de la Universitat de les Illes Balears. Este estudio también presenta los factores estresores más determinantes en el aumento de estos niveles así como las estrategias de afrontamiento más utilizadas. Adicionalmente, hemos examinado la relación existente entre los niveles de estrés y el expediente académico. Este estudio contribuye al conocimiento científico sobre el estrés y el rendimiento académico, incluyendo cuatro hallazgos especialmente importantes. Primero, las estudiantes universitarias presentan niveles de estrés académico más elevados que sus homólogos masculinos. Segundo, "las evaluaciones de los profesores" y "el tipo de trabajo que piden los profesores" son los estímulos estresores que muestran una diferencia estadísticamente significativa cuando se analiza en función del género, percibiendo mayor estrés por parte de las universitarias. Tercero, las mujeres utilizan estadísticamente más "la ventilación y confidencias" como estrategia de afrontamiento en comparación con el género masculino. Cuarto, no hay relación significativa entre el estrés académico y el rendimiento académico. Es importante tener en cuenta que en este estudio se describen las diferencias observadas en los universitarios de las Islas Baleares, y los datos no son, por tanto, representativos para otras poblaciones.

\section{Niveles de estrés académico}

Los elevados niveles de estrés académico encontrados en nuestro trabajo están en concordancia con los obtenidos en otros estudios mediante el cuestionario SISCO (Barraza, 2008; de La Rosa-Rojas et al., 2015; Mejía-Rubalcava, Alanís-Tavira, Argueta-Figueroa y Legorreta-Reyna, 2012; Oliván Blázquez, Boira Sarto y López del Hoyo, 2011). Estos niveles, pueden ser explicados en parte, por "la sobrecarga de tarea y trabajos escolares", así como por "las evaluaciones de los profesores". Paralelamente parece ser, que existen otras variables moduladoras psicológicas y psicosociales, y no controladas en este estudio, que podrían explicar los elevados niveles de estrés 
académico; entre otras estas pueden ser el apoyo social y los factores de personalidad (Baqutayan, 2011; Hystad, Eid, Laberg, Johnsen y Bartone, 2009). Las diferencias detectadas en función del género son consistentes con las obtenidas en otros estudios (Feldman, Goncalves, Chacón-Puignau, Zaragoza y Pablo, 2008; García-Ros, PérezGonzález, Pérez-Blasco y Natividad, 2012). Estas diferencias pueden ser debidas a que las mujeres admitan que determinadas situaciones les generan estrés, que les provocan un elevado impacto emocional y que, por tanto, les resultan más difíciles de afrontar (García-Ros et al., 2012). Por otro lado y según Barra-Almagia (2009) las mujeres adolescentes, frente a los varones, manifiestan niveles superiores de percepción del estrés y ánimo depresivo, argumentos que pueden contribuir a la explicación de las mencionadas diferencias.

\section{Factores estresores}

Podemos afirmar que los estresores académicos que más percepción de estrés causan, en los universitarios incluidos en el presente trabajo, son "la sobrecarga de tarea y trabajos escolares" y "las evaluaciones de los profesores". Esto coincide con los datos aportados por la literatura científica previa, que indicaba que la evaluación y la sobrecarga de trabajo son las dimensiones que generan mayor estrés académico (Cabanach et al., 2010; Spangler, Pekrun, Kramer y Hofmann, 2002). El carácter estresor de las pruebas de evaluación parece ser que presenta un origen multicausal, siendo la aprensión a la evaluación, el miedo al fracaso y ciertos elementos de naturaleza cognitiva los motivos que expliquen esta condición (Dyson y Renk, 2006). Aunque tradicionalmente se ha vinculado la sobrecarga al número de horas que el estudiante debe dedicar a la asignatura, existen otras variables como las características de los contenidos, la dificultad de las actividades, la falta de información y las relaciones de carácter bidireccional que deben ser consideradas para la explicación de este estresor académico (Kember, 2004). Las estudiantes universitarias presentan niveles de estrés significativamente más elevados en las dimensiones de "las evaluaciones de los profesores" y el "tipo de trabajo que piden los profesores", respecto a los varones. Una posible explicación podría encontrarse en el hecho de que las mujeres prestan mayor atención a las emociones que los varones, mientras que éstos informan de una mayor capacidad para reparar sus estados emocionales (Extremera Pacheco y Durán Durán, 2007). Algunos trabajos plantean la posibilidad de que los estudiantes universitarios varones sean más reticentes a informar sobre su nivel de estrés e incluso sean menos conscientes de su estado (Davies et al., 2000), aspecto que podría explicar que las puntuaciones de los diversos estresores académicos sean inferiores respecto a las mujeres.

\section{Estrategias de afrontamiento}

La evidencia sugiere que la elección de ciertas estrategias de afrontamiento puede tener un efecto protector ante los efectos negativos del estrés académico. Las estrategias de resolución de problemas o de búsqueda de información son conductas activas de afrontamiento que se asocian, más frecuentemente, con una mayor competencia y un funcionamiento positivo (de La Rosa-Rojas et al., 2015). Por el 
contrario, el uso de estrategias centradas en la adopción de estrategias de evitación se relaciona con menores niveles de bienestar psicológico y la presencia de una mayor afectividad negativa (Chao, 2011). Nuestros datos sugieren que las estrategias de afrontamiento más utilizadas son "elaboración de un plan y ejecución de sus tareas" y "concentrarse en resolver la situación que me preocupa", ambas implican una conducta activa. Nuestros resultados se muestran en la misma línea que los presentados en otros estudios (de La Rosa-Rojas et al., 2015; Oliván Blázquez et al., 2011). Según los datos de nuestro trabajo la estrategia de "ventilación y confidencias" es más utilizada significativamente por las mujeres que por los hombres. Estos datos son contradictorios con los de Barraza (2008) quien no encontró significación alguna. Aunque según Brougham, Zail, Mendoza, y Miller (2009) las mujeres utilizan, con mayor frecuencia, estrategias centradas en el control de las emociones. Argumento que corrobora nuestros resultados y reafirma la evidencia científica previa (Eaton y Bradley, 2008; Stanton, Kirk, Cameron y Danoff-Burg, 2000).

\section{Estrés académico y rendimiento académico}

Un gran número de trabajos han revelado una relación negativa entre estrés y rendimiento académico, es decir, que los alumnos que presentan índices elevados de estrés académico son los que obtienen peores calificaciones (Lumley y Provenzano, 2003; Sohail, 2013; Struthers, Perry y Menec, 2000; Zajacova, Lynch yEspenshade, 2005). Sin embargo, la actual evidencia científica presenta cierta controversia. Feldman et al., (2008) encontraron que el nivel de estrés académico influía en el rendimiento de los varones, pero no en el de las mujeres. Los resultados de nuestro trabajo no muestran ninguna relación entre el nivel de estrés y el rendimiento académico incluso después de analizar esta interacción a través del género. Estas diferencias entre estudios puede explicarse, en parte, porque la disminución del rendimiento académico no depende tanto de los estresores académicos sino más bien de las estrategias de afrontamiento que se poseen (Ciarrochi, Deane y Anderson, 2002). Esta controversia es posible que también encuentre un razonamiento en la existente diferencia de género en el funcionamiento cognitivo-emocional en el ámbito académico y de patrones atribucionales (Feldman et al., 2008). Aunque las mujeres tengan un nivel de estrés académico más elevado que los varones, presentan un manejo más eficiente del tiempo, priorizan metas, planean y se organizan mejor (Misra, Crist y Burant, 2003) y al contrario de lo que pudiera esperarse esta mayor percepción de estrés no se acompaña con un descenso de su rendimiento académico (Pomerantz, Altermatt y Saxon, 2002).

\section{Fortalezas y limitaciones}

El presente estudio tiene ciertas limitaciones que deben reconocerse. El instrumento SISCO, utilizado para evaluar el nivel de estrés, los factores estresores y los estilos de afrontamiento es autoreportado, además el estudio es de corte-transversal y no permite la evaluación de las relaciones encontradas. Asimismo, es importante mencionar que existen otros factores que podrían influir en el estrés académico, tales como las características sociales, económicas y culturales que no fueron controladas 
en este estudio. A pesar de estas limitaciones, este estudio ha encuestado a un $25 \%$ de los estudiantes del Grado en Educación Primaria, ofreciendo una muestra suficientemente representativa para poder presentar conclusiones con cierta contundencia. Otra fortaleza de este trabajo es la diferenciación por género de los resultados, aportando evidencia a la escasa literatura al respecto (Barraza, 2008; Echavarri, Godoy y Olaz, 2007; Feldman et al., 2008). Hay cierta evidencia que relaciona mayor percepción de estrés con un mejor rendimiento académico, pero por otro lado, algunas investigaciones constatan que elevados niveles de estrés se vinculan a niveles de rendimiento inferiores. Esta controversia sustenta la necesidad de profundizar en la investigación entre estrés y rendimiento académico, así como ampliar la evaluación de los niveles de estrés en momentos cruciales del curso académico y controlar indicadores de rendimiento académico que puedan influir (tasa de abandono, tasa de no presentados, duración de los estudios) en estas relaciones.

\section{Conclusiones}

Teniendo en cuenta la información mostrada, los resultados del presente estudio indican que los niveles de estrés académico en estudiantes del Grado en Educación Primaria de la Universitat de les Illes Balears están influenciados por el género, siendo las mujeres las que perciben mayor estrés que los hombres, aunque ambos presentan niveles medios-altos. Los estresores académicos no difieren en función del género, pero por el contrario sí que se observan niveles más elevados en casi todas las dimensiones en el género femenino. Pudiéndose concluir que las mujeres presentan mayor vulnerabilidad delante de los estresores académicos, lo que podría explicarse por su mayor preocupación por agradar a los adultos o por su forma de aproximarse y enjuiciar la evaluación (Cabanach, Fariña, Freire, González y Ferradás, 2013). Los estudiantes, tanto hombres como mujeres, responden de forma convergente a los estresores académicos, aunque con ligeras diferencias. Las mujeres universitarias han mostrado mayores niveles en las estrategias de afrontamiento centradas en la expresión de sentimientos y en la búsqueda de apoyo social (dimensión "ventilación y confidencias"), en comparación con sus compañeros varones. Tanto niveles de estrés muy bajos como niveles elevados se han correlacionado con niveles de rendimiento académico inferiores; nuestros datos muestran que los niveles de estrés no influyen negativamente en el rendimiento académico. Por lo tanto, atendiendo al elevado nivel de estrés académico, es recomendable la implementación de programas de intervención orientados hacia la prevención de éste y así fomentar la capacidad de los estudiantes para soportar situaciones de estrés académico.

\section{REFERENCIAS BIBLIOGRÁFICAS}

Backovi, D. V., Živojinovi , J. I., Maksimovi, J. y Maksimovi, M. (2012). Gender differences in academic stress and burnout among medical students in final years of education. Psychiatria Danubina, 24(2), 175-181.

Baqutayan, S. (2011). Stress and social support. Indian Journal of Psychological Medicine, 33(1), 29-34. http://doi.org/10.4103/0253-7176.85392. 
Barra-Almagia, E. (2009). Influencia del estrés y el ánimo depresivo sobre la salud adolescente: analisis concurrente y prospectivo. Universitas Psychologica, 8(1), 175-182.

Barraza, A. (2007). Propiedades psicométricas del Inventario SISCO del estrés académico. Revista Psicologia Científica, 9(10). Recuperado de http://www.psicologiacientifica.com/sisco-propiedades-psicometricas.

Barraza, A. (2008). El estrés académico en alumnos de maestría y sus variables moduladoras: Un diseño de diferencia de grupos. Avances en Psicologia Latinoamericana, 26(2), 270-289.

Bayram, N. y Bilgel, N. (2008). The prevalence and socio-demographic correlations of depression, anxiety and stress among a group of university students. Social Psychiatry and Psychiatric Epidemiology, 43(8), 667-672. http://doi.org/10.1007/ s00127-008-0345-x.

Brougham, R. R., Zail, C. M., Mendoza, C. M. y Miller, J. R. (2009). Stress, sex differences, and coping strategies among college students. Current Psychology, 28(2), 85-97. http://doi.org/10.1007/s12144-009-9047-0.

Cabanach, R., Fariña, F., Freire, C., González, P. y Ferradás, M. M. (2013). Diferencias en el afrontamiento del estrés en estudiantes universitarios hombres y mujeres. European Journal of Education and Psychology, 6(1), 19-32.

Cabanach, R., Fernández-Cervantes, R., González, L. y Freire, C. (2010). Estresores académicos percibidos por estudiantes universitarios de ciencias de la salud. Fisioterapia, 32(4), 151-158. http://doi.org/10.1016/j.ft.2010.01.005.

Chambel, M. J. y Curral, L. (2005). Stress in academic life: Work characteristics as predictors of student well-being and performance. Applied Psychology: An International Review, 54(1), 135-147.

Chao, R. C.-L. (2011). Managing Stress and Maintaining Well-Being: Social Support, Problem-Focused Coping, and Avoidant Coping. Journal of Counseling \& Development, 89(3), 338-348. http://doi.org/10.1002/j.1556-6678.2011.tb00098.x.

Ciarrochi, J., Deane, F. P. y Anderson, S. (2002). Emotional intelligence moderates the relationship between stress and mental health. Personality and Individual Differences, 32(2), 197-209. http://doi.org/10.1016/S0191-8869(01)00012-5.

Davies, J., McCrae, B. P., Frank, J., Dochnahl, A., Pickering, T., Harrison, B., ... Wilson, K. (2000). Identifying male college students' perceived health needs, barriers to seeking help, and recommendations to help men adopt healthier lifestyles. Journal of American College Health, 48(6), 259-67. http://doi. org/10.1080/07448480009596267.

de La Rosa-Rojas, G., Chang-Grozo, S., Delgado-Flores, L., Oliveros-Lijap, L., Murillo-Pérez, D., Ortiz-Lozada, R. y Yhuri Carreazo, N. (2015). Level of stress and coping strategy in medical students compared with students of other careers. Gaceta Médica de México, 151(4), 443-449.

Dyson, R. y Renk, K. (2006). Freshmen adaptation to university life: depressive symptoms, stress, and coping. Journal of Clinical Psychology, 62(10), 1231-44. http://doi.org/10.1002/jclp.20295.

Eaton, R. J. y Bradley, G. (2008). The role of gender and negative affectivity in stressor appraisal and coping selection. International Journal of Stress Management, 15(1), 94-115. http://doi.org/10.1037/1072-5245.15.1.94. 
Echavarri, M., Godoy, J. C. y Olaz, F. (2007). Diferencias de género en habilidades cognitivas y rendimiento académico en estudiantes universitarios. Universitas Psychologica, 6(2), 319-329.

Ekpenyong, C. E., Daniel, N. E. y Aribo, E. O. (2013). Associations between academic stressors, reaction to stress, coping strategies and musculoskeletal disorders among college students. Ethiopian Journal of Health Sciences, 23(2), 98-112.

Extremera Pacheco, N. y Durán Durán, A. (2007). Inteligencia emocional y su relación con los niveles de burnout, engagement y estrés en estudiantes universitarios. Revista de Educación, 342, 239-256. http://doi.org/10.4321/S113212962006000200006.

Feldman, L., Goncalves, L., Chacón-Puignau, G., Zaragoza, N. y Pablo, J. De. (2008). Relaciones entre estrés académico, apoyo social, salud mental y rendimiento académico en estudiantes universitarios venezolanos. Universitas Psychologica, 7(3), 739-752.

García-Ros, R., Pérez-González, F., Pérez-Blasco, J. y Natividad, L. A. (2012). Evaluación del estrés académico en estudiantes de nueva incorporación a la universidad. Revista Latinoamericana de Psicología, 44, 143-154.

Hamaideh, S. H. (2012). Gender differences in stressors and reactions to stressors among Jordanian university students. The International Journal of Social Psychiatry, 58(1), 26-33. http://doi.org/10.1177/0020764010382692.

Hystad, S. W., Eid, J., Laberg, J. C., Johnsen, B. H. y Bartone, P. T. (2009). Academic Stress and Health: Exploring the Moderating Role of Personality Hardiness. Scandinavian Journal of Educational Research, 53(5), 421-429. http://doi. org/10.1080/00313830903180349.

Kember, D. (2004). Interpreting student workload and the factors which shape students' perceptions of their workload. Studies in Higher Education, 29(2), 165184. http://doi.org/10.1080/0307507042000190778.

Keogh, E., Bond, F. W. y Flaxman, P. E. (2006). Improving academic performance and mental health through a stress management intervention: Outcomes and mediators of change. Behaviour Research and Therapy, 44(3), 339-357. http://doi. org/10.1016/j.brat.2005.03.002.

Lue, B.-H., Chen, H.-J., Wang, C.-W., Cheng, Y. y Chen, M.-C. (2010). Stress, personal characteristics and burnout among first postgraduate year residents: a nationwide study in Taiwan. Medical Teacher, 32(5), 400-407. http://doi. org/10.3109/01421590903437188.

Lumley, M. A. y Provenzano, K. M. (2003). Stress management through written emotional disclosure improves academic performance among college students with physical symptoms. Journal of Educational Psychology, 95(3), 641-649. http://doi.org/10.1037/0022-0663.95.3.641.

Mejía-Rubalcava, C., Alanís-Tavira, J., Argueta-Figueroa, L. y Legorreta-Reyna, A. (2012). Academic stress as a risk factor for dental caries. International Dental Journal, 62(3), 127-31. http://doi.org/10.1111/j.1875-595X.2011.00103.x.

Misra, R., Crist, M. y Burant, C. J. (2003). Relationships Among Life Stress, Social Support, Academic Stressors, and Reactions to Stressors of International Students in the United States. International Journal of Stress Management, 10(2), 137-157. http://doi.org/10.1037/1072-5245.10.2.137. 
Ojeda Pérez, B., Ramal López, J., Calvo Francés, F. y Vallespín Montero, R. (2001). Estrategias de afrontamiento al estrés y apoyo social. Psiquis, 22(3), 155-168.

Oliván Blázquez, B., Boira Sarto, S. y López del Hoyo, Y. (2011). Estrés y otros factores psicológicos asociados en estudiantes de fisioterapia. Fisioterapia, 33(1), 19-24. http://doi.org/10.1016/j.ft.2010.12.002.

Orlandini, A. (1999). El estrés, que es y como evitarlo. España: Fondo de Cultura Económica.

Park, J., Chung, S., An, H., Park, S., Lee, C., Kim, S. Y. y Kim, K.-S. (2012). A structural model of stress, motivation, and academic performance in medical students. Psychiatry Investigation, 9(2), 143-9. http://doi.org/10.4306/pi.2012.9.2.143.

Pérez, M., Martín, A., Rodríguez, F., Borda, M. y Del Río, C. (2003). Estrés y Rendimiento Académico en Estudiantes Universitarios. Cuadernos de Medicina Psicosomática Y Psiquiatría de Enlace, 67/68.

Pomerantz, E. M., Altermatt, E. R. y Saxon, J. L. (2002). Making the grade but feeling distressed: Gender differences in academic performance and internal distress. Journal of Educational Psychology, 94(2), 396-404. http://doi.org/10.1037/00220663.94.2.396.

Regehr, C., Glancy, D. y Pitts, A. (2013). Interventions to reduce stress in university students: a review and meta-analysis. Journal of Affective Disorders, 148(1), 1-11. http://doi.org/10.1016/j.jad.2012.11.026.

Salanova-Soria, M., Martínez-Martínez, I., Bresó-Esteve, E., Llorens-Gumbau, S. y Grau-Gumbau, R. (2005). Bienestar psicológico en estudiantes universitarios: facilitadores y obstaculizadores del desempeño académico. Anales de Psicología, 21(1), 170-180.

Selye, H. (1946). The general adaptation syndrome and the diseases of adaptation. The Journal of Clinical Endocrinology and Metabolism, 6, 117-230. http://doi. org/10.1210/jcem-6-2-117.

Sohail, N. (2013). Stress and academic performance among medical students. Journal of the College of Physicians and Surgeons-Pakistan : JCPSP, 23(1), 67-71. http:// doi.org/01.2013/JCPSP.6771.

Spangler, G., Pekrun, R., Kramer, K. y Hofmann, H. (2002). Students' Emotions, Physiological Reactions, and Coping in Academic Exams. Anxiety, Stress \& Coping, 15(4), 413-432. http://doi.org/10.1080/1061580021000056555.

Stanton, A L., Kirk, S. B., Cameron, C. L. y Danoff-Burg, S. (2000). Coping through emotional approach: scale construction and validation. Journal of Personality and Social Psychology, 78(6), 1150-1169. http://doi.org/10.1037/00223514.78.6.1150.

Struthers, C., Perry, R. y Menec, V. (2000). An examination of the relationship among academic stress, coping, motivation, and performance in college. Research in Higher Education, 41(5), 581-592. http://doi.org/10.1023/A:1007094931292.

Struthers, C. W., Perry, R. P. y Menec, V. H. (2000). An Examination of the Relationship Among Academic Stress, Coping, Motivation, and Performance in College. Research in Higher Education, 41(5), 581-592. http://doi.org/10.2307/40196403.

Zajacova, A., Lynch, S. M. y Espenshade, T. J. (2005). Self-efficacy, stress, and academic success in college. Research in Higher Education, 46(6), 677-706. http://doi.org/10.1007/s11162-004-4139-z. 